\title{
Characterisation and deterioration of mineral papers
}

\section{Cancy Chu \& Petronella Nel}

Grimwade Centre for Cultural Materials Conservation, School of Historical and Philosophical Studies, The University of Melbourne, Melbourne, Australia, VIC, 3010

Cancy Chu * corresponding author

Email: cancyc@student.unimelb.edu.au

ORCID: http://orcid.org/0000-0002-2173-261X

Petronella Nel

ORCID: http://orcid.org/0000-0002-8509-547X

This is the author accepted manuscript. The version of record is published by Taylor \& Francis as:

Chu, C \& Nel, P 2019, 'Characterisation and deterioration of mineral papers', AICCM Bulletin,

vol. 40, no. 1, pp. 37-49, https://doi.org/10.1080/10344233.2019.1672951. 


\section{Characterisation and deterioration of mineral papers}

Mineral paper, also known as rich mineral paper, is a paper-like material manufactured from calcium carbonate with a small amount of high-density polyethylene (HDPE), instead of traditional cellulose-based fibres. For environmental reasons, mineral paper was designed to degrade when exposed to sunlight. It was the aim of this study to address the research gap in conservation literature describing the properties and degradation patterns of mineral paper. Three mineral paper samples were characterised using visual examination techniques and analysed using Fourier transform infrared spectroscopy with attenuated total reflectance (ATRFTIR) and scanning electron microscopy with energy dispersive X-ray spectroscopy (SEMEDS). Calcium carbonate, talc, kaolin, high-density polyethylene and an unknown trace material(s) were identified in these mineral papers. Under accelerated ageing conditions, the mineral paper samples consistently demonstrated a higher rate of chemical and physical degradation compared to a cellulose paper standard when exposed to visible light and ultraviolet radiation. Through this, a greater understanding was obtained of mineral paper composition, its ageing trajectory, and response to environmental factors. Further research is required to identify the unknown trace element(s) and whether photo-sensitive additives are present. These results should help to inform the identification, storage, display and treatment of mineral paper-based collections.

Keywords: paper degradation; stone paper; synthetic paper; FTIR spectroscopy; SEM-EDS; colour spectrophotometry, photodegradation

\section{Introduction}

\section{Background}

Mineral paper is a synthetic, paper-like material, mainly composed of ground calcium carbonate $\left(\mathrm{CaCO}_{3}\right)$ obtained from industrial stone waste and a small amount of high-density polyethylene (HDPE) (Hol 2013, p. 6). Mineral paper, also known as stone paper, rich mineral paper, rock paper, limestone paper or eco-paper, closely resembles conventional cellulose paper in appearance and function. However, technically it is not a true paper, as it does not contain cellulose-based pulped fibres (Hunter 1978, p. 5; Hubbe 2016, p. 5656). Furthermore, mineral paper can be distinguished from most other synthetic papers by its high mineral content. Calcium carbonate acts as the base 
component of mineral paper, rather than being added as an opacity-inducing filler for a synthetic polymer base (Mustalish 2007, p. 143). Mineral paper is waterproof, printable, writable, and includes a plastic component like many other synthetic papers. Since its invention in the late 1990s, mineral paper has become commercially available in the form of waterproof books, notebooks and artists' paper. Conservators, curators, artists, and others may find themselves in need of information regarding the composition and ageing properties of mineral papers when designing guidelines regarding its use, storage and display.

\section{History of synthetic and mineral paper}

Since the late twentieth century, the paper industry has tailored synthetic papers such as Teslin ${ }^{\circledR}$ and Polyart $^{\circledR}$ to specific purposes through the careful selection of polymers, additives and coatings (Katz 2017). Synthetic papers can be more suitable than cellulose papers for certain applications such as printed currency and labels, as they can be made waterproof, ultraviolet (UV) resistant, and compatible with the recyclability of plastic bottles and other labelled items (Larson 2006). Currently, synthetic papers are mostly manufactured with polypropylene, with a minority of papers made out of polyethylene, polyester and polystyrene (Mustalish 2007, p. 144).

Building on developments from the 1960s, Taiwan Lung Meng Technology Co., Ltd. (TLM) was the first to commercially manufacture mineral paper in 1998 (Hsieh et al. 2013, p. 320; Affeldt, Leung \& Yang 2016, p. 5). Mineral paper is so named for its paper-like form and use of limestone as its main raw material. This recently invented mineral paper is distinct from historical stone paper printing matrices, which were coated cellulose-based papers used in the early nineteenth century by artists as an experimental replacement for lithographic stones (Taylor 2018). Mineral paper is marketed with a high environmental sustainability focus, claiming a low carbon footprint, tree-less and water-less production, recyclability, and 'complete' degradability, thus minimising its landfill contribution (Lu, Wang \& Wang 2011; Nuco 2013; Gaia-kraft 2015). To date, mineral paper has been internationally 
distributed under trade names such as Repap ${ }^{\circledR}$, FiberStone ${ }^{\circledR}$, TerraSkin ${ }^{\mathrm{TM}}$, Parax ${ }^{\mathrm{TM}}$, Rockstock ${ }^{\mathrm{TM}}$ and Karst Stone Paper ${ }^{\mathrm{TM}}$.

Table 1. Composition of mineral paper according to product distributors (D), manufacturer (M) and sustainability reports $(\mathrm{R})$, quoting percentages where available.

\begin{tabular}{|c|c|c|c|c|}
\hline \multirow{2}{*}{$\begin{array}{l}\text { Reference } \\
\text { Karst Stone Paper }(2018, \text { p. 3) }\end{array}$} & \multicolumn{2}{|c|}{ Type Inorganic Component } & \multirow{2}{*}{$\frac{\text { Organic Component }}{10 \% \text { non-toxic resin }}$} & \multirow{2}{*}{$\frac{\text { Additives }}{\text { No mention }}$} \\
\hline & $\mathrm{D}$ & $80-90 \% \mathrm{CaCO}_{3}$ & & \\
\hline Manifesto Brands (c. 2013) ${ }^{a}$ & $\mathrm{D}$ & $\mathrm{CaCO}_{3}$ & HDPE/PE/non-toxic resin & No mention \\
\hline Nuco International Ltd. (2013, p. 1) & $\mathrm{D}$ & $80 \% \mathrm{CaCO}_{3} /$ chalk & $20 \% \mathrm{HDPE}$ & None \\
\hline Parax Paper (c. 2013) & $\mathrm{D}$ & $80 \% \mathrm{CaCO}_{3} /$ limestone & $20 \% \mathrm{HDPE}$ & None \\
\hline The Stone Paper Co. (c. 2010) & $\mathrm{D}$ & Up to $81 \% \mathrm{CaCO}_{3}$ & About $18 \%$ PE & No mention \\
\hline Gaia-kraft (2015) & $\mathrm{D}$ & $\mathrm{CaCO}_{3}$ & HDPE & No mention \\
\hline TLM (c. 2012) & M & $60-80 \% \mathrm{CaCO}_{3}$ & $20-40 \%$ non-toxic resin & Coating \\
\hline $\operatorname{Hol}(2013, \text { p. } 5)^{b}$ & $\mathrm{R}$ & $80 \% \mathrm{CaCO}_{3}$ & $18 \% \mathrm{HDPE}$ & $2 \%$ proprietary coating \\
\hline Affeldt, Leung \& Yang $(2016, \text { p. } 25)^{b}$ & $\mathrm{R}$ & $73 \% \mathrm{CaCO}_{3}$ & $20 \% \mathrm{HDPE}$ & $4 \%$ proprietary additives, $3 \%$ ink \\
\hline
\end{tabular}

${ }^{a}$ References presented provide specifications for the same TLM-manufactured products, except for the Manifesto Brands entry, which is the distributor for Repap ${ }^{\circledR}$, discontinued in 2007.

${ }^{b}$ Percentages calculated by weight, not directly quoted.

\section{Composition and manufacture of mineral paper}

Unlike cellulose and synthetic papers, the main ingredient of mineral paper is ground calcium carbonate $\left(\mathrm{CaCO}_{3}\right)$, sourced from raw limestone and chalk obtained as waste from stone quarries and construction offcuts (Tang 2010, p. 43; Ezcurra 2012, p. 164; Hol 2013, p. 6). Recycled high-density polyethylene (HDPE) and surface coatings are added to bind the material and enhance printability respectively, resulting in a product that is waterproof and physically durable. Mineral paper is usually reported by mineral paper distributors to consist of $60-90 \%$ calcium carbonate and $10-40 \%$ HDPE, while sustainability reports and manufacturers additionally document coatings and printing inks (Table 1).

The production process of mineral paper is described by the TLM manufacturer website and third-party studies (TLM c. 2012; Hol 2013, p. 6; Affeldt, Leung \& Yang 2016, p. 12). After 
grinding $\mathrm{CaCO}_{3}$ mineral into a fine powder, it is combined with recycled HDPE pellets and proprietary additives under high heat to form small composite pellets. Next, the pellets are extruded into a continuous sheet according to the thickness required, and coated with a proprietary material on both sides to enhance printability. Lastly, the roll is cut into usable sheets or rolls and packaged for further production, such as printing and final distribution. Remaining offcuts and scraps are retained and re-pelletised.

\section{Degradation of mineral paper}

Mineral paper's short lifespan when exposed to environmental elements is widely acknowledged in its promotional media, although the reputed timeframe for natural breakdown ranges from six to eighteen months (Tang 2010, p. 43; Zero Waste Europe 2011; Manifesto Brands c. 2013). The following quote describes the ageing behaviour of Repap ${ }^{\circledR}$ according to its distributor Manifesto Brands:

If REPAP is left outside and left exposed to continuous UV light, then after approximately 14-18 months, it will become brittle and break itself down like an egg shell. The calcium carbonate, returns to the earth naturally [sic]. Like traditional paper and when used in a traditional notebook sense (carried in a bag or stored closed on a desk or shelf), REPAP will not discolour, turn brittle or decompose. However, we do not say REPAP is archival. (Manifesto Brands c. 2013, penultimate paragraph)

Prolonged exposure to sunlight causes mineral paper to turn brittle, crack like an 'egg shell', and degrade into a powder, giving it a distinctive deterioration process compared to other papers. Its unusual degradation has been observed in studies where mineral paper was purposefully exposed to environmental factors: one a natural ageing study by forensic scientists (Elliot \& Stadmeyer 2012), and one an artificial ageing study by a student conservator (Kuypers 2016). These studies highlight the unusual ageing behaviour of mineral paper compared to other synthetic and cellulose papers. 


\section{Uses of mineral paper}

Due to its tear-resistant and waterproof properties, mineral paper has been used in a variety of printed products where moisture resistance or tensile strength is a beneficial feature. Many mineral paper suppliers list paper grades suitable for printed commercial products, such as posters and banners, food and manufacturer labels, tickets, and shopping and gift bags (The Stone Paper Co. c. 2010; Nuco 2013; Heart Stone Paper c. 2017). Mineral paper has been used in published books, particularly ones wishing to demonstrate a commitment to environmentally sustainable ideals (Braungart \& McDonough 2002; Lin 2011). Mineral paper journals, sketch-pads and plain paper products have been produced for office printing, writing, artwork, and other applied media. Increasingly, mineral paper notebooks can be purchased from ordinary bookstores and stationery suppliers, under names such as Karst Stone Paper ${ }^{\mathrm{TM}}$, Heart Stone Paper ${ }^{\mathrm{TM}}$, Oxford ${ }^{\mathrm{TM}}$, and Nu Stone $^{\mathrm{TM}}$. A successfully crowd-funded planner branded Hustle ${ }^{\mathrm{TM}}$ demonstrates the popular appeal of spill-proof and environmentally-minded mineral paper products (Hustle Stone Paper 2016). Mineral paper products have been used as an artist's material for sketching and watercolour painting. Artists such as Julio Reyes (2017), Taylor Ikin (2013), and Tina Mammosa (2013) have documented their experiences using mineral paper in their artworks, describing how the surface allows for artistic experimentation and novel media application techniques.

\section{Experimental Methodology}

\section{Research aim}

Considering that mineral paper is currently used as a medium for writing, printing and artwork, caretakers of cultural collections may soon encounter mineral paper objects in need of appropriate storage, display or other conservation expertise. This study aimed to determine the composition and ageing properties of mineral papers through materials characterisation and artificial ageing techniques of multiple mineral paper samples. 
Table 2. Mineral paper samples (S) and standards (C) selected for experiments.

\begin{tabular}{|c|c|c|}
\hline Sample & Product & Source \\
\hline $\mathrm{S} 1$ & Nu Stone ${ }^{\mathrm{TM}}$ nu: Tradie Waterproof Notebook, A5 ruled pages & Officeworks, Bourke St \\
\hline $\mathrm{S} 2$ & Luckies $^{\mathrm{TM}}$ The Waterproof Notebook No. 02, A6 blank pages & Readings, Lygon St \\
\hline $\mathrm{S} 3$ & Ogami ${ }^{\circledR}$ Professional Notebook, $90 \times 140 \mathrm{~mm}$ ruled pages & Pen City, Elizabeth St \\
\hline $\mathrm{C} 1^{a}$ & Whatman ${ }^{\circledR}$ filter paper $41,150 \mathrm{~mm}$ diameter circles (cellulose standard) & Grimwade Centre, Swanston St \\
\hline $\mathrm{C} 2^{b}$ & Riverina 2L milk carton, recycling code 2 (HDPE standard) & Grimwade Centre, Swanston St \\
\hline $\mathrm{C} 3^{b}$ & Chem-Supply Calcium carbonate LR CL009-500G ( $\mathrm{CaCO}_{3}$ standard) & Grimwade Centre, Swanston St \\
\hline $\mathrm{C} 4^{b}$ & $\begin{array}{l}\text { Chem-Supply Talc Purified TG Hydrous Magnesium Silicate TT043-500G } \\
\text { (Talc standard) }\end{array}$ & Grimwade Centre, Swanston St \\
\hline $\mathrm{C} 5^{b}$ & Ajax Chemicals Kaolin acid washed technical D3247 (Kaolin standard) & Grimwade Centre, Swanston St \\
\hline
\end{tabular}

${ }^{a}$ Artificial ageing control

${ }^{b}$ FTIR standard

\section{Materials}

Three mineral paper notebook samples (S1-3) were purchased from stationers and bookshops in Melbourne, Victoria in April 2017 (Table 2). Samples were selected for their availability in Australia and representation of the cost range of commercially available mineral paper notebooks. Standards for cellulose paper (Whatman), HDPE, cotton wool and common paper fillers $\mathrm{CaCO}_{3}$ (Chem-Supply), talc (Ajax) and kaolin (Chem-supply) (C1-5 respectively) were obtained for FTIR spectra comparison. Whatman ${ }^{\circledR}$ filter paper was selected for the cellulose paper standard (C1) due to its wide use in conservation, known ageing properties, and availability in the testing location.

\section{Sample preparation for artificial ageing}

S1-3 and C1 were prepared for artificial ageing. Samples were cut into rectangular strips with a ruler and scalpel, maintaining consistency of grain direction with reference to the spine of the book. Sample size (Table 3) was selected based on equipment availability and size constraints.

\section{Sample preparation for scanning electron microscopy (SEM)}

S1-3 and C1 were prepared by cutting into approximately $1 \mathrm{~cm}^{2}$ pieces. Samples were mounted on carbon tape on a glass slide, and coated with a thin layer of carbon to increase electrical conductivity. 
Table 3. Sample sizes of four accelerated ageing tests, limited by ageing apparatus.

\begin{tabular}{llll}
\hline Test & Length $(\mathbf{c m})$ & Width $\mathbf{( c m )}$ & Relative size \\
\hline Daylight (DA) & 7 & 3 & Full page \\
Ultraviolet (UV) & 7 & 1.5 & Half page \\
Thermal (TH) & 3.5 & 1.5 & Quarter page \\
Relative humidity (RH) & 3.5 & 1.5 & Quarter page \\
\hline
\end{tabular}

Table 4. Conditions of four accelerated ageing tests.

\begin{tabular}{llllll}
\hline Test & Radiation & Temp. $\left({ }^{\circ} \mathbf{C}\right)$ & $\mathbf{R H}^{a}$ & Duration & Sampling frequency \\
\hline Daylight (DA) & Fluorescent light bank, 14000 & 22 & $38 \%$ & 6 weeks & 1 week \\
& lux, UV at $72 \mu \mathrm{W} / \mathrm{lumen}$ & & & & \\
Ultraviolet (UV) & UV A at $340 \mathrm{~nm}, 1.2 \mathrm{~W} / \mathrm{m}^{2}$ & 60 & $30 \%$ & 4 weeks & Varied $^{b}$ \\
Thermal (TH) & Darkness & 60 & $10 \%$ & 4 weeks & Varied $^{b}$ \\
Relative humidity (RH) & Darkness & 60 & $90 \%$ & 4 weeks & Varied $^{b}$ \\
\hline
\end{tabular}

${ }^{a}$ Relative humidity was measured at the beginning of the experiment with an ELSEC 764UV+ environmental monitor.

${ }^{b}$ Refer to Table 5 for sampling times.

\section{Artificial ageing}

Ageing protocol was modelled on a previous ageing experiment by Kuypers (2016), which simulated ageing using four environments in order to isolate the effect of light, ultraviolet $\left(\mathrm{UV}_{\mathrm{A}}\right)$ radiation, heat and relative humidity. Samples S1-3 and C1 were aged under daylight simulation (DA) for up to 6 weeks, and for up to 4 weeks for elevated $U_{V}$ radiation (UV), elevated temperature (TH), and elevated relative humidity $(\mathrm{RH})$ (Table 4). Due to the high intensity of $\mathrm{UV}_{\mathrm{A}}$ exposure in the UV experiment, and as changes were observed in a mineral paper sample after the first week of ageing in Kuyper's experiment (2016, p. 71), sampling occurred more frequently over the first week to observe any point of reaction time change (Table 5). As the TH and RH experiments acted as controls for the UV experiment, these were sampled at the same frequency as for the UV experiment. 
Table 5. Sampling times for ageing experiments

\begin{tabular}{llc}
\hline Test & Hours aged & $168,336,504,672,840,1008$ \\
\hline $\mathrm{DA}^{a}$ & $2,4,8,12,16,20,24,48,72,96,120,144,168,336,504,672$ \\
$\mathrm{UV}, \mathrm{TH}, \mathrm{RH}^{a, b}$ & 2 \\
\hline
\end{tabular}

${ }^{a} \mathrm{DA}=$ Daylight, $\mathrm{UV}=$ Ultraviolet, $\mathrm{TH}=$ Thermal, $\mathrm{RH}=$ Relative humidity.

${ }^{b}$ Sampling occurred more frequently in first week to observe any rapid changes in this period.

Daylight (DA). Six sets of full-sized samples were placed flat on a sheet of blue archival board and aged in a light bank powered by eight parallel Philips CS TLD 36W/840 cool white fluorescent tubes fixed at $30 \mathrm{~cm}$ above the samples. Even illumination was ensured with an ELSEC 754UV+ monitor. One set of samples was removed each week over a period of 6 weeks.

Ultraviolet $\left(U V_{A}\right)$. Sixteen sets of half-sized samples were placed in eight sample holders of a UV ageing instrument (Q-Lab QUV Accelerated Weathering Tester, Model QUV/se with solar irradiance control) with $340 \mathrm{~nm}$ light tubes, set at $1.2 \mathrm{~W} / \mathrm{m}^{2}$ and $60^{\circ} \mathrm{C}$. Cycle time was set at 168 hours (7 days) and restarted each week. One set of samples was removed at each sampling time shown in Table 5.

Thermal (TH). Sixteen sets of quarter-sized samples were sealed in two polypropylene containers (Décor Microsafe ${ }^{\circledR}$ Oblong 3.5L) and placed on steaming racks to ensure air circulation. Containers were placed in a laboratory oven (Laboratory Equipment PTY LTD 'LABEC', Model 0DW24) set at $60^{\circ} \mathrm{C}$. One set of samples was removed at each sampling time shown in Table 5.

Relative humidity $(R H)$. Experimental set-up was identical to $\mathrm{TH}$ experiment, except that two $25 \mathrm{~mL}$ beakers of deionised water were refilled weekly to the $20 \mathrm{~mL}$ mark and placed in the centre of each container. 


\section{Characterisation and analysis}

\section{Visual examination}

Visual examination under ambient light conditions was used to document the appearance, texture, tearing and folding behaviours, and thickness of unaged mineral paper samples. Visual information was collected to describe optical properties traditionally used for cellulose-based paper (Britt 2012), as conventions for mineral paper examination have not been established. Colour, gloss, opacity and texture were evaluated through visual examination and manual handling, and were recorded descriptively. Folding and tear tests were used to determine the presence of a grain direction (Balloffet \& Hille 2005, p. 78). Thickness was measured with a Mercer Type 58 thickness gauge and averaged over three readings. Transmitted and raking light applied with a handheld LED torch, together with magnification with a handheld USB digital microscope (no brand) at 20x magnification were used to observe the surface morphology and consistency of the papers. Samples were examined using reflected short- $(254 \mathrm{~nm})$ and long- $(365 \mathrm{~nm})$ wave UV radiation to determine the presence of optical brighteners. Lastly, reaction of the papers to the application of water-based Uni-ball Signo UMR-100 0.5 pen with UMR-5 ink, and a HB Staedtler graphite pencil were recorded. Samples were submerged in a room temperature water bath for 30 minutes before and after media application, as an indication of waterproofness. However, no testing standards were followed for waterproof testing.

After ageing, samples were visually examined and evaluated for signs of degradation. In particular, colour change, flexibility, and shape distortion were the focus of attention. Changes were evaluated with careful handling and bending of samples. Samples were documented using digital photography (Nikon D3300 DSLR camera).

\section{Colour spectrophotometry}

A colour spectrophotometer (BYK-Gardner spectro-guide sphere gloss) set to D65 and $10^{\circ}$ illumination was used to quantify the colour difference between the aged and unaged samples. 
Calibration of the device was carried out before each measurement session. An average reading of $\Delta \mathrm{E}_{00}$ in CIE 2000 colour space was determined from 10 averaged measurements recorded 10 seconds between each reading, ensuring a standard deviation of below 0.01 .

\section{Infrared spectroscopy}

Fourier transform infrared spectroscopy with attenuated total reflection (ATR-FTIR) was used to identify the polymer composition of the mineral paper samples before ageing, and to observe chemical changes after ageing. A Bruker Alpha-P FTIR spectrometer equipped with a diamond ATR window (Bruker Optik GmbH, Ettlingen, Germany) was used to record spectra of the S1-3 and C1 before and after four weeks of ageing. Standards C2-6 were also analysed for comparison. All spectra were recorded in the spectral range of 4000-400 cm-1 with 32 co-added scans at a spectral resolution of $4 \mathrm{~cm}^{-1}$. Before each measurement, the diamond ATR crystal was cleaned with isopropanol, a cleanness test performed, and a background measurement collected. Data acquisition and processing was performed with OPUS software (version 7.5, Bruker Optik GmbG, Ettlingen, Germany).

\section{Scanning electron microscopy}

Scanning electron microscopy with energy-dispersive X-ray spectroscopy (SEM-EDS) was used to identify the elements and observe the physical structure of the unaged papers. A Philips XL30 ESEM was used to conduct a Backscattered Electron (BSE) scan of the surfaces of the three carbon-coated samples at $1500 \mathrm{x}$ magnification and $10.0 \mathrm{kV}$ accelerated voltage. SEM-EDS elemental spectra of the three samples were then taken to identify the elemental composition of different areas identified in the BSE scan. 


\section{Results}

\section{Characterisation}

\section{Visual examination}

Under visual examination, the three unaged mineral papers were similar in appearance and physical properties. S1, S2 and S3 had a uniform, white, matte, opaque appearance under ambient reflected light. Samples had a homogenous surface texture with a slight tooth on both sides. When handled, samples felt smooth and were noticeably heavier than expected of either paper or plastic. When torn, samples stretched before breaking, similar to a polyethylene sheet. Tearing showed a preferential direction, similar to a grain in cellulose papers. Samples tore with minimal resistance and in a smooth line parallel to the spine (with the grain), and gave a more ragged, scallop-like edge when torn perpendicular to the spine (against the grain). However, a similar directional preference was not noticeable for folding. Thickness averages were $0.13,0.13$ and $0.12 \mathrm{~mm}$ for $\mathrm{S} 1,2$ and 3 respectively.

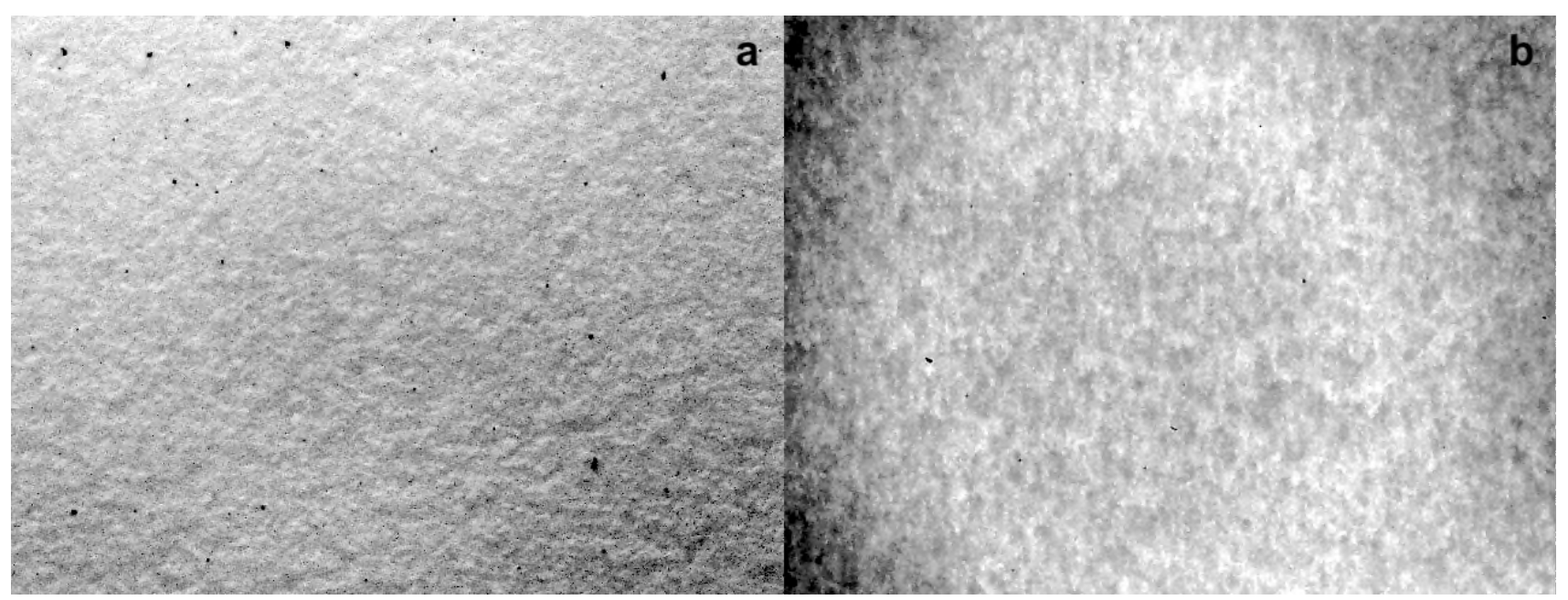

Figure 1. S2 under (a) raking and (b) transmitted LED light, captured with a 5 MP digital USB microscope, 20x magnification.

When observed under 20x magnification, randomly scattered black inclusions were seen embedded in the white surface, with a noticeably higher number appearing in S2 than for the other two samples. For the two samples with printed lines (S1 and S3), the ink lines in S1 were much 
darker, thicker and more embedded than the lines in S3. A rough surface was observed with raking light (Figure 1a). Subtle variations in consistency were observed under transmitted light and magnification, which indicate a directionality of film extrusion, although no fibres were observed (Figure 1b).

Samples were impervious to water. They showed no swelling, weakening or other signs of absorption after 30 minutes of immersion. Samples resumed original texture and appearance once water was wiped off with a paper towel and did not cockle after drying. When dry, samples accepted media application from a water-based ink and graphite pencil. Water-based ink began to bleed once submerged in water, although remained legible after 30 minutes. Water did not appear to affect the graphite.

No fluorescence was observed under either short- or long-wave UV radiation indicating that optical brighteners are not part of the formulation.

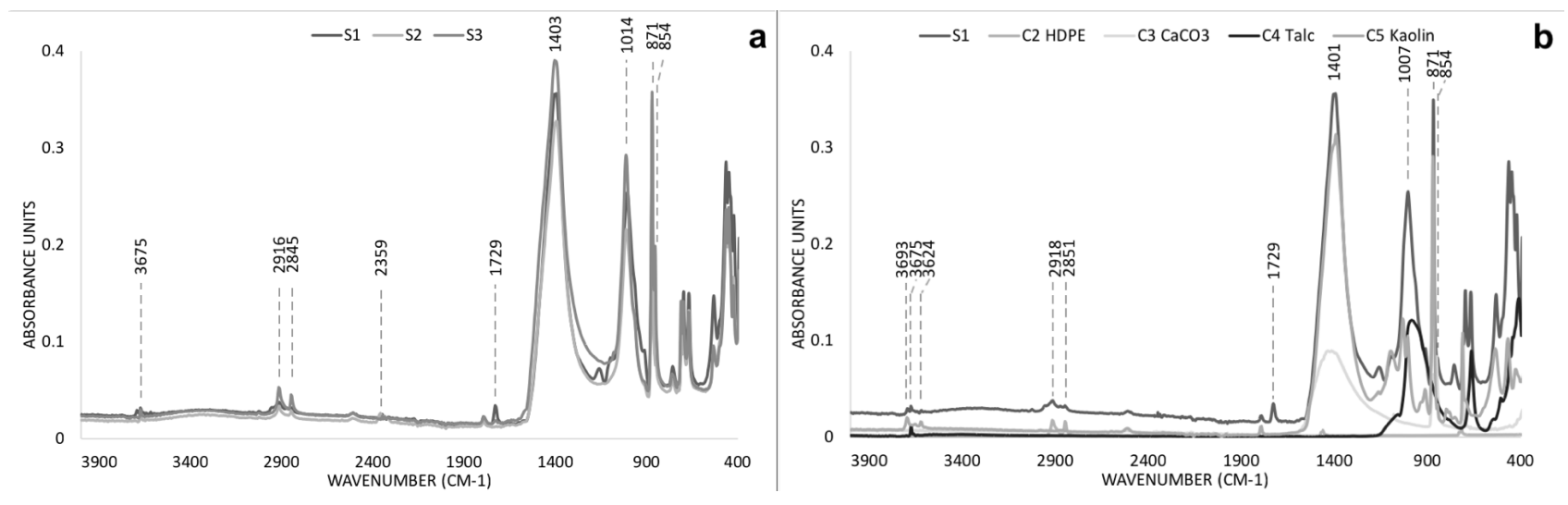

Figure 2. Overlaid FTIR spectra showing (a) similarity between $\mathrm{S} 1, \mathrm{~S} 2$ and $\mathrm{S} 3$ over range $4000-400 \mathrm{~cm}^{-1}$ and (b) scaled spectra of $\mathrm{S} 1$ compared to standards $\mathrm{C} 2$ : $\mathrm{HDPE}, \mathrm{C} 3: \mathrm{CaCO}_{3}, \mathrm{C} 4$ : Talc and C5: Kaolin.

\section{Infrared spectroscopy}

FTIR spectra of mineral paper samples were very similar to each other (Figure 2a). All three samples share the majority of peaks in both the functional group and fingerprint regions. A spectrum 
search of the OPUS 7.5 Bruker Reference Library returned a highest match of $63.2 \%$ similarity with ground calcium carbonate, a result which was disregarded due to the low similarity value. Sample IR spectra were visually compared to IR spectra of known standards of $\mathrm{HDPE}, \mathrm{CaCO}_{3}$, kaolin, talc and cotton (Figure 2b). Sample peaks matched spectra of $\mathrm{HDPE}, \mathrm{CaCO}_{3}$, kaolin and talc, but not cotton. Peaks were assigned based on matches with characteristic absorption peaks of known standards (Stuart 2007, p. 123; Chércoles et al. 2009, p. 2087; Derrick, Stulik \& Landry 1999, pp. 194-195):

- A strong, broad peak at $1588-1170 \mathrm{~cm}^{-1}$ correlates with the $\mathrm{O}-\mathrm{H}$ bending in kaolin, or $\mathrm{CO}_{3}$ stretching in $\mathrm{CaCO}_{3}$;

- A strong, broad peak at $1072-905 \mathrm{~cm}^{-1}$ correlates with the asymmetric Si-O-Si stretching in kaolin and talc;

- A strong, narrow peak at $871 \mathrm{~cm}^{-1}$ matches Si-O stretching in kaolin;

- A strong, narrow peak at $854 \mathrm{~cm}^{-1}$ correlates with $\mathrm{O}-\mathrm{C}-\mathrm{O}$ bending in $\mathrm{CaCO}_{3}$. A weak, narrow peak at $3674 \mathrm{~cm}^{-1}$ correlates with $\mathrm{O}-\mathrm{H}$ bending in talc;

- Weak, narrow peaks between 2920 and $2840 \mathrm{~cm}^{-1}$ correlate with $\mathrm{CH}_{2}$ stretching in HDPE;

- $\mathrm{S} 1$ additionally has weak, narrow peaks at 3691 and $3622 \mathrm{~cm}^{-1}$ that correlate with $\mathrm{O}-\mathrm{H}$ stretching found in kaolin (Derrick, Stulik \& Landry 1999, pp. 194-195); and

- A presence of unmatched peaks at $1729 \mathrm{~cm}^{-1}$ in $\mathrm{S} 1$ and $2362 \mathrm{~cm}^{-1}$ in $\mathrm{S} 2$ suggest the inclusion of unknown compounds in the mineral paper samples.

Results are summarised in Table 6.

\section{Scanning electron microscopy}

Backscattered electron (BSE) scans of S1, S2 and S3 revealed a similar appearance and physical structure. Three distinct features were observed under 1500x magnification (Figure 3): 
Table 6. Major FTIR absorption peak assignments of mineral paper samples (Derrick, Stulik \& Landry 1999, pp. 194-195; Stuart 2007, p. 123; Chércoles et al. 2009, p. 2087).

\begin{tabular}{llll}
\hline Sample $^{a}$ & Peak position & Assignment & Conjectured Components \\
\hline S1 & 3691,3620 & O-H stretching & Kaolin \\
S1, S2, S3 & 3675 & O-H stretching & Talc \\
S1, S2, S3 & 2916,2849 & $\mathrm{CH}_{2}$ stretching & HDPE \\
S2 & 2359 & Unassigned & Unknown \\
S1 & 1729 & Unassigned & Unknown \\
S1, S2, S3 & $1588-1174$ & $\mathrm{CO}_{3}$ stretching and O-H bending & $\mathrm{CaCO}_{3}, \mathrm{Kaolin}$ \\
S1, S2, S3 & $1072-905$ & Asymmetric Si-O-Si stretching & $\mathrm{Kaolin}_{\text {Talc }}$ \\
S1, S2, S3 & 871 & Si-O stretching & $\mathrm{Kaolin}$ \\
S1, S2, S3 & 854 & O-C-O bending & $\mathrm{CaCO}_{3}$ \\
\hline
\end{tabular}

${ }^{a}$ Refer to Table 2 for sample descriptions.

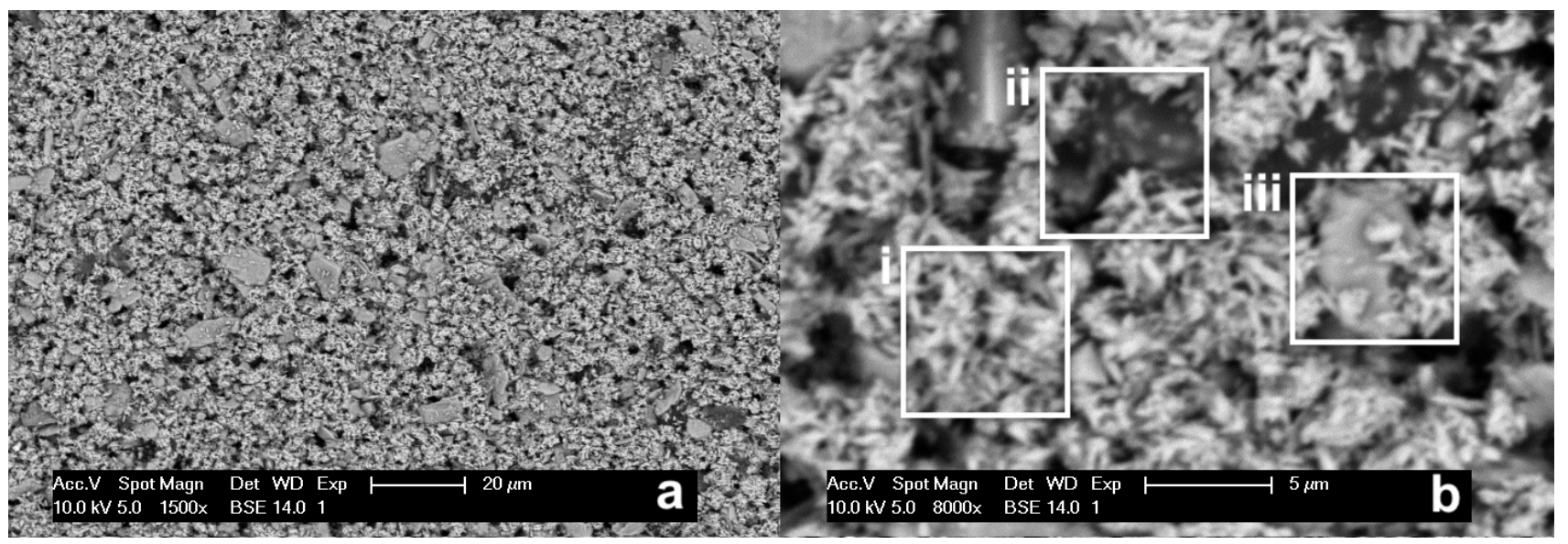

Figure 3. Detection of Backscattered Electrons (BSE) of S2, captured with Philips XL30 ESEM, 10.0kV accelerated voltage, showing (a) overall structure at 1500x magnification, and (b) detail of (i) bright needles, (ii) amorphous clusters and (iii) defined grains at 8000x magnification.

- Small, bright, needle-like clusters

- Large, bright to dark, well-defined grains

- Dark, amorphous-shaped clusters

Each of the three features were analysed via EDS to determine their elemental composition (Table 7). 
Table 7. Summary of elements detected with SEM/EDS of mineral paper samples ( $\mathrm{S}=$ strong signal, $\mathrm{t}=$ trace $)$.

\begin{tabular}{llllllll}
\hline Area, Sample & H & C & O & Ca & Mg & Al & Si \\
\hline Needles, S1, S2 & S & S & S & S & t & & T \\
Grain S1 & S & t & S & & & S & S \\
Grain S2 & S & S & S & & S & S \\
Grain S3 & S & t & S & & S & S \\
Amorphous S1 & S & S & S & t & & \\
Amorphous S2 & S & S & t & t & & \\
Area scan, 5x5 microns, S3 & S & S & S & S & S & & S \\
\hline
\end{tabular}

Needle-like clusters. A predominant feature across all three samples were small, needle-like clusters that appeared bright under BSE detection. These needle-like shapes were particularly well-defined for S3. Elements detected for the needles are hydrogen, carbon, oxygen and calcium, with trace amounts of magnesium and silicon. The abundance of this feature and its heavy composition, indicated by relative brightness under BSE, suggest that these are calcium carbonate particles, mixed with an organic material such as HDPE.

Defined grains. Embedded in the needles were large, bright to dark grains with well-defined edges. In addition to carbon and hydrogen, the large grains included the metals aluminium and silicon in S1, and aluminium and magnesium in S2 and S3. The large grains in S1 match the composition of kaolin, $\mathrm{Al}_{2} \mathrm{Si}_{2} \mathrm{O}_{5}(\mathrm{OH})_{4}$. The large grains in $\mathrm{S} 2$ and $\mathrm{S} 3$ match the composition of talc, $\mathrm{Mg}_{3} \mathrm{Si}_{4} \mathrm{O}_{10}(\mathrm{OH})_{2}$.

Amorphous clusters. Further embedded in the surface were dark, amorphous areas with irregular edges and varying sizes. Elements detected for these areas were mainly carbon and hydrogen, with trace amounts of oxygen and calcium. A dark appearance indicates a composition of lower atomic number, likely to be an organic material such as HDPE. 




Figure 4. Comparison of four-week aged (a) $\mathrm{C} 1$ and (b) S1, captured with a Nikon D3300 camera, with 18$55 \mathrm{~mm}$ VR II lens, at $40 \mathrm{~mm}$ zoom, f-stop 8, and shutter speed 1/200s. C1 is relatively stable, with noticeable discolouration of the RH-aged sample only. S1 displays discolouration in DA, UV and RH-aged samples, along with severe fragmentation of UV-aged samples.

\section{Artificial ageing}

\section{Visual examination}

Examination of the physical and visual properties of the aged samples indicated that the main signs of degradation were yellow-brown discolouration and embrittlement. Embrittlement, as established with handling and bending, increased in severity over time; with the most severe cases showing complete fragmentation after only light handling. S1, S2 and S3 showed signs of deterioration at a faster rate than the $\mathrm{C} 1$ (Figure 4). Mineral paper embrittlement occurred most severely in $\mathrm{UV}_{\mathrm{A}}$-aged samples. By the end of the four-week UV experiment, S1, S2 and S3 showed advanced embrittlement, fragmenting upon removal from the sample holder, while the cellulose paper sample remained stable. Initial fragmentation occurred at five days for S1 and six days for S2. S3 transitioned more sharply than the other two samples, going from no fracturing to overall fracturing at the two-week point. Overall fragmentation, meaning when resulting pieces were smaller than approximately $1 \mathrm{~cm}^{2}$, occurred around two weeks for all three mineral paper samples. No directional preference was observed in the fragmentation patterns. Although overall fragmentation is the most advanced observed sign of embrittlement, a decrease in flexibility and strength can also be observed when handling the papers before fractures occur. Warping of edges was observed in some $\mathrm{UV}_{\mathrm{A}}$-aged samples, but not consistently. Embrittlement and warping were not observed for the DA, TH, and 
RH-aged samples, although the texture of the TH-aged mineral papers became rougher to touch.

Significant discolouration was observed in both DA and $\mathrm{UV}_{\mathrm{A}}$-aged samples, and to a lesser extent for the $\mathrm{RH}$-aged samples. A yellow-brown tone increased in $\mathrm{UV}_{\mathrm{A}}$-aged mineral paper samples up to approximately four days, after which the level of discolouration remained relatively constant.

Discolouration increased until about four weeks in the DA experiment, although the level of discolouration was much lower than that of $\mathrm{UV}_{\mathrm{A}}$-aged samples for $\mathrm{S} 1$ and $\mathrm{S} 2$. Mineral paper samples from TH experiments were relatively unchanged, showing similar appearance to the unaged samples. RH-aged cellulose paper samples also showed signs of colour change, but other cellulose paper samples remained stable.

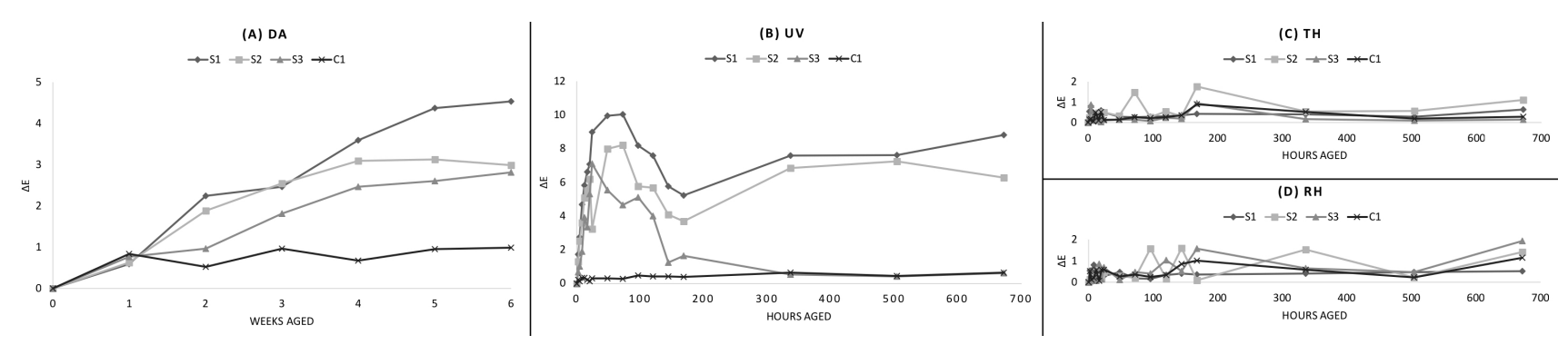

Figure 5. Graphs of $\Delta \mathrm{E}_{00}$ in $\mathrm{S} 1, \mathrm{~S} 2, \mathrm{~S} 3$ and $\mathrm{C} 1$ vs duration aged of four accelerated aging experiments, showing increasing colour change over time. $\mathrm{C} 1$ comparatively yields insignificant results.

\section{Colour spectrophotometry}

An overall pattern of increasing colour change is clearly seen in the graphs of $\Delta \mathrm{E}_{00}$ versus time of aging (Figure 5). For S1 and S2, resulting colour change is much higher for the $\mathrm{UV}_{\mathrm{A}}$-aged mineral paper samples than in the other three experiments, as seen from a $\Delta \mathrm{E}_{00}$ of approximately 10 in $\mathrm{UV}, 4$ in DA and less than 1 for TH and RH after ageing for four weeks (504 hours). Results for C1 consistently show a $\Delta \mathrm{E}_{00}$ of less than 1.5 , considered an indicator of visually noticeable colour change (Pretzel 2008, p. 762), except in the samples aged for four weeks in the RH experiment.

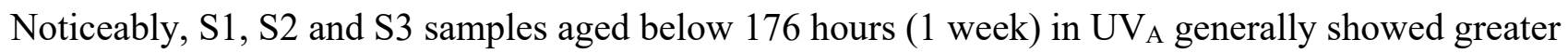


colour change than those aged for four weeks. Due to instrument complications, these samples were not aged continuously, but for two to three disjointed periods to provide the cumulative exposure time. Resulting frequent temperature changes may have increased the rate of colour change in the samples aged in the first week. An alternate explanation is that the continued exposure to $U_{\mathrm{A}}$ radiation induced a photo-bleaching effect (see Discussion).

From the $\Delta \mathrm{E}_{00}$ results, the contribution of $\mathrm{UV}_{\mathrm{A}}$ radiation to the colour change of mineral paper is clearly observed. As a small portion of the radiation in the visual light bank is in the $\mathrm{UV}_{\mathrm{A}}$ region, the fact that samples from the DA experiment show the second-highest rate of degradation is consistent with the theory that $\mathrm{UV}_{\mathrm{A}}$ is the greatest contributor to mineral paper colour change. Contribution of the elevated temperature of $60^{\circ} \mathrm{C}$ in the $\mathrm{UV}_{\mathrm{A}}$ ageing machine can be ruled out by the $60^{\circ} \mathrm{C} \mathrm{TH}$ and $\mathrm{RH}$ results. Samples from $\mathrm{TH}$ and $\mathrm{RH}$ experiments conducted at $60^{\circ} \mathrm{C}$ in the dark show negligible colour change over the course of the experiment. Considering the stability of the samples aged under the elevated relative humidity of the RH experiment, the results rule out a hydrolysis degradation pathway. Based on the pattern of $\Delta \mathrm{E}_{00}$ values under $\mathrm{UV}_{\mathrm{A}}$ conditions, photo-oxidation is the most likely pathway of mineral paper degradation.

\section{Infrared spectroscopy}

At four weeks of ageing, S1, S2 and S3 aged in the UV and DA experiments show a decrease in absorption for the two weak C-H peaks around 2919 and $2849 \mathrm{~cm}^{-1}$, the strong broad peaks at 15901170 and 1070-900, strong narrow peak at $871 \mathrm{~cm}^{-1}$ (Figure 6a), suggesting breakage of previously detected bonds. In comparison, little change is observed for the thermal (TH and RH) experiments and for $\mathrm{C} 1$ across all experiments.

Smoothing of the two broad, weak functional group peaks at 2916 and $2849 \mathrm{~cm}^{-1}$, correlated with $\mathrm{CH}_{2}$ stretching, suggests the degradation of HDPE, which may be related to chain scission resulting from photo-oxidation. No formation of oxidation products (Chew, Gan \& Scott 1977) 
occurred in the carbonyl $(\mathrm{C}=\mathrm{O}) 1780-1650 \mathrm{~cm}^{-1}$ or hydroxyl $(\mathrm{OH}) 3700-3400 \mathrm{~cm}^{-1}$ regions, though they were expected. This may be due to the small amount of HDPE in the samples. A decrease in absorption of the strong IR peaks at 1590-1170, 1070-900, and $871 \mathrm{~cm}^{-1}$ correlates with Si-O-Si stretching of kaolin and $\mathrm{CO}_{3}$ stretching in $\mathrm{CaCO}_{3}$, Si-O-Si stretching of kaolin and talc, and the Si-O stretching of kaolin at $873 \mathrm{~cm}^{-1}$ (Figure $6 \mathbf{b}$ ) respectively. This indicates the degradation of the coating and filling materials used in the mineral papers. It also confirms the presence of kaolin in S2 and S3, which was previously inconclusive.


Figure 6. Overlaid FTIR spectra of unaged and 4-week aged S1, showing (a) detail of 3000-2800 $\mathrm{cm}^{-1}$ range showing smoothing of peaks associated with $\mathrm{CH}_{2}$ stretching in DA and UV experiments, and (c) detail of 1600$1200 \mathrm{~cm}^{-1}$ range showing smoothing of peak associated with $\mathrm{CO}_{3}$ stretching and $\mathrm{O}-\mathrm{H}$ bending in DA and UV experiments. 


\section{Discussion}

\section{Characterisation}

Mineral papers were characterised using visual analysis, ATR-FTIR and SEM-EDS to reveal their physical and chemical properties. Findings from visual examination are very similar to the waterproof and paper-like properties described in mineral paper marketing media. Like cellulose paper, mineral paper accepts both wet and dry media applications. Unlike cellulose paper, mineral papers were not affected by exposure to water, and do not contain visible fibres at 20x magnification. A directional preference for tearing was identified, which may be explained by the direction of extrusion. For all visual examination tests, the three mineral paper samples behaved very similarly. The greatest differences lay in the appearance of the printed ink, which varied between the S1 and S3 samples, and the identification of kaolin suggested by the presence of aluminium in S1 but not S2 or S3 with SEM-EDS. This may be due to variations in ink absorbance or surface printing preparation, which may be explained by their differing manufacturers.

Papers were examined under reflected short- and long-wave UV radiation, and no visual fluorescence was observed for any samples. This indicates a lack of optical brighteners (Grant 2000, p. 2). A previous UV fluorescence study observed small, dark inclusions in TerraSkin ${ }^{\mathrm{TM}}$, but not the rest of the paper (Ezcurra 2012, p. 165). While similar dark inclusions were observed for the three samples under magnification, they did not fluoresce for the three selected samples.

ATR-FTIR and SEM-EDS analysis indicate mineral papers most likely contain HDPE, chalk $\left(\mathrm{CaCO}_{3}\right)$, talc $\left(\mathrm{Mg}_{3} \mathrm{Si}_{4} \mathrm{O}_{10}(\mathrm{OH})_{2}\right)$, and kaolin $\left(\mathrm{Al}_{2} \mathrm{Si}_{2} \mathrm{O}_{5}(\mathrm{OH})_{4}\right)$, with some additional unidentified material(s) in small quantities. As $\mathrm{CaCO}_{3}$, talc and kaolin are common paper coating materials (Hubbe \& Gill 2016, p. 2887), their strong absorption under FTIR relative to HDPE and abundance under SEM indicates that the analytical methods are limited to the surface of the paper substrate. Furthermore, the presence of unidentified components indicates the inclusion of materials not present in the tested standards. Further analysis with gas chromatography-mass spectroscopy (GC-MS) may 
assist with identifying the unknown material(s), particularly if plasticisers and/or additives are present (eds. Lavédrine, Fournier \& Martin 2012, p. 105). Repeating analysis after removing the surface layer of the papers may also reveal compositional differences in the main substrate.

\section{Degradation}

Samples of three mineral papers (S1-3) and one control cellulose paper (C1) were artificially aged in four accelerated ageing experiments: UV, DL, TH, and RH. Mineral papers consistently showed significantly higher rates of physical degradation in $\mathrm{UV}_{\mathrm{A}}$ and simulated daylight experiments compared to the cellulose paper standard. As similar levels of ageing were not observed under elevated temperature and relative humidity conditions, the findings are consistent with a photocatalysed oxidation degradation path. These findings correlate with the study by Kuypers (2016), in which $\mathrm{UV}_{\mathrm{A}}$-aged Repap mineral paper sample disintegrated after two weeks. The 'eggshell-like' description used by Kuypers and mineral paper marketing is a highly appropriate description of the embrittlement and fragmentation resulting from $U_{\mathrm{A}}$ ageing. Notably, Kuypers' elevated thermal experiments resulted in significant discolouration of mineral paper samples, a result which was not reproduced in this set of experiments. This may be explained by the fact that the current experiment was conducted $60^{\circ} \mathrm{C}$ rather than $100^{\circ} \mathrm{C}$.

Early signs of mineral paper deterioration were identified as yellow discolouration and embrittlement. Next, increasing embrittlement and warping of the edges may occur, finally leading to widespread fragmentation. Fragmentation was aggravated with handling, progressing the paper from a warped and cracked state to disintegration into small, friable pieces. Although testing was conducted without applied media, it is obvious that any supported image or information would be lost over time. As samples tested were very small, testing of larger samples may reveal differing warping and fragmentation patterns. 
Overall colour spectrophotometry results show the comparative colour stability of cellulose paper over mineral paper samples, particularly under UV and DA conditions. However, the results for UV-aged samples do not show a direct correlation between colour change and hours aged. UVaged mineral paper samples displayed an increasing colour change up to approximately $100 \mathrm{~h}$, then a decrease in colour change up to $200 \mathrm{~h}$, and finally plateau leading up to $700 \mathrm{~h}$. This may be explained by an initial yellowing of samples due to chemical degradation, followed by a photobleaching effect from UV exposure. A similar phenomenon has been observed in weathered wood, where lignin on the surface is broken down by sunlight, causing an initial yellowing effect, but then pales when degradation products are leached through water contact (Feist \& Hon 1984, pp. 411414). The reasons for the decrease in colour change between 100 and $200 \mathrm{~h}$ may be revealed with further examination of the surface chemical changes. However, ATR-FTIR results did not reveal any further understanding of this effect.

ATR-FTIR analysis identified peaks correlating with disruption of $\mathrm{HDPE} \mathrm{CH}_{2}$ bonds, which further support a photo-oxidative pathway. However, the formation of carbonyl oxidation products at $\sim 1700 \mathrm{~cm}^{-1}$ was not observed. As the contribution of the elevated temperature at $60{ }^{\circ} \mathrm{C}$ during the $\mathrm{UV}$ ageing experiment is ruled out by the $\mathrm{TH}$ and $\mathrm{RH} 60^{\circ} \mathrm{C}$ ageing experiments, embrittlement is most likely caused by exposure to $\mathrm{UV}_{\mathrm{A}}$ radiation. ATR-FTIR analysis of daylight and UV-aged mineral paper samples also found a decrease in Si-O bonds associated with kaolin and talc composites at $\sim 1000$ and $871 \mathrm{~cm}^{-1}$. Changes in these bonds may be associated with dehydration reactions. However, dehydration is often only observed in reactions catalysed by high temperatures (Bellotto et al. 1994), which would have affected samples aged in elevated temperature settings more than in simulated daylight ageing. Further research is necessary to determine the nature of $U_{\mathrm{A}}$ and light-induced degradation of the silicate components in mineral paper.

Resource limitations resulted in the omission of some appropriate analytical techniques, such as tensile strength testing of aged papers (Karlovits \& Gregor-Svetec 2012; Nicomrat \& Tharajak 
2017), standardised waterproof testing (ASTM E96 / E96M-16), and microscopy for the examination of the physical appearance of mineral papers after ageing. As all samples have been retained, further testing may be practical, but may be limited by the size of samples needed for tensile strength tests.

The present study does not purport to represent the ageing timeframe of real mineral paper collections under ambient conditions, due to the use of artificial ageing techniques. However, based on the intensity of light exposure during the DA experiment, and the experimental result that all three samples exceeded a $\Delta \mathrm{E}_{00}$ value of 1.5 after 3 weeks, the following theoretical approximation of time before noticeable discolouration was calculated. Given 50 lux of light exposure for $7 \mathrm{~h}$ a day, 312 days a year, the mineral papers tested would start discolouring after the equivalent of 65 years (tested light intensity $\times$ duration of testing for noticeable colour change $=1.4 \times 10^{4} \operatorname{lux} \times 504 \mathrm{~h}=7.1 \times 10^{6}$ lux-hours; years of 50 lux exposure $=7.1 \times 10^{6}$ lux-hours / (50 lux x 7 h/day x 6 days/week x 52 weeks/year) $=65$ years). For the sake of comparison, if the exposure were increased to 200 lux, used for moderately sensitive objects in museums (Slade 2003, p. 2), the time decreases to 16 years. Depending on the conditions of storage and display used, some mineral paper works may already be in need of conservation attention. Artist Julio Reyes displays mineral paper artworks sealed in frames with UV-resistant glazing and avoiding direct sunlight (Reyes 2017). A future project could include an accelerated ageing test of the mineral paper when shielded by UV resistant glass or glazing, to determine the effectiveness of such a preservation method. It could also be argued that the thermal ageing should be conducted over a longer time period before significant changes will be observed.

Lastly, the development of mineral paper treatment options is an open field of research. Waterproof properties of mineral paper may be incompatible with traditional paper treatments as they rely on water-based adhesives or solvent delivery systems. Investigation of suitable adhesives for mineral paper may be of use to conservators needing to repair future mineral paper collections. 


\section{Recommendations}

Although the long-term behaviour of mineral paper in real ageing conditions is not known, the use of mineral papers for archival purposes is not recommended based on artificial ageing tests. Mineral paper is designed to be photodegradable. Based on artificial ageing tests, conservators should consider mineral paper to have a medium sensitivity to light, like colour photographs, and a very high sensitivity to UV, as with newsprint (Michalski 2018). Therefore, the amount of acceptable change should be assessed when forming a conservation strategy.

To minimise change, mineral papers should be stored and displayed with minimal exposure to light and sources of UV radiation, ideally at exposures of 50 lux and $75 \mu \mathrm{W} /$ lumen or below, respectively. For example, short-term display in areas lit at 50 lux or below with IR-and UV-filtered light, and storage in darkness, is recommended. Additionally, storage environments for mineral paper collections in stable condition should follow the AICCM Environmental Guidelines for Australian Cultural Heritage Collections (2018) of $15-25^{\circ} \mathrm{C}$, and $40-60 \%$ or $45-65 \%$ relative humidity for temperate climates or subtropical and tropical climates respectively, in order to minimise environmental fluctuations and avoid catalysing colour change. If embrittlement or warping of mineral paper has occurred, handling should be minimised to prevent cracking and fragmentation. If yellowing or embrittlement has occurred, migration of the information through means such as digitisation should be carried out as soon as possible before further distortion or destruction of supported information occurs. Artists and other potential users should be informed of the light sensitivity of mineral paper when considering its use in cultural media. Conservators may additionally consider accepting and managing the eventual change of this sensitive material through practices such as documentation, migration and reiteration.

\section{Conclusion}

Three brands of mineral papers were characterised using visual examination, digital microscopy, SEM-EDS, and ATR-FTIR analysis. Mineral paper is distinct from cellulose papers due to its 
waterproof properties and lack of cellulose fibres. Mineral papers are stated to contain calcium carbonate (60-90\%) and HDPE (40-10\%) (TLM c. 2012). SEM-EDS and ATR-FTIR analysis confirmed the presence of $\mathrm{CaCO}_{3}$ and HDPE, and found evidence that they also contain talc, kaolin, and other unidentified material(s). Findings indicate the presence of previously unknown and unadvertised materials included in mineral paper, which are likely to be proprietary print coatings. Results demonstrate that ATR-FTIR and SEM-EDS can be complementarily used to identify unknown coating materials in paper materials.

The same three mineral papers and a cellulose paper control were artificially aged under four accelerated ageing conditions (visible, $\mathrm{UV}_{\mathrm{A}}$, thermal and thermal with $\mathrm{RH}$ ). Visual photography, colour spectrophotometry and ATR-FTIR analysis were applied to document and evaluate deterioration. Results indicate that mineral paper is sensitive to $\mathrm{UV}_{\mathrm{A}}$ radiation and visible light. Deterioration appears to follow a photo-oxidation pathway, resulting in discolouration, embrittlement, warping and fragmentation of the mineral papers. Thus, $\mathrm{UV}_{\mathrm{A}}$ protection is critical for this type of paper.

\section{Acknowledgements}

The authors are grateful to the Grimwade Centre at the University of Melbourne for facilitating the project, Graham Hutchinson at the University of Melbourne School of Earth Sciences for technical assistance with the SEM instrument, and Albertine Hamilton and Marika Kocsis at State Library Victoria for valuable discussions on visual examination. This research was supported by the Australian Government through the Australian Research Council's Linkage Project funding scheme (project LP160100160) and the University of Melbourne Faculty of Arts Research and Graduate Studies scheme (RAGS). The views expressed herein are those of the authors and are not necessarily those of the Australian Government, the Australian Research Council or the University of Melbourne.

\section{Author Biographies}

Cancy $\mathrm{Chu}$ is a $\mathrm{PhD}$ student at the University of Melbourne researching conservation approaches for plastics in archives. She has completed a MA of Cultural Materials Conservation (2017) specialising in works on paper at the University of Melbourne, and a BA (2015) majoring in Studio Art at Reed College, Portland, 
Oregon. Her current research is part of an Australian Research Council (ARC) Linkage Project led by Dr Petronella Nel, and is supported by the Australian Commonwealth Government's Research Training Program and the University of Melbourne's Melbourne Research Scholarship.

Dr Petronella Nel is a Senior Lecturer at the Grimwade Centre for the Conservation of Cultural Materials of the University of Melbourne. She has a BSc (Honours, 1990) in Chemistry, a PhD in Chemistry (2000) and an MA in Cultural Materials Conservation (2006), from the University of Melbourne. She is leading a collaborative ARC Linkage Project 'A national framework for managing malignant plastics in Museum Collections'. She is interested in developing analytical techniques for characterising materials in order to inform their preservation.

\section{References}

Affeldt, C, Leung, A \& Yang, K 2016, 'Life cycle assessment of stone paper, polypropylene film, and coated paper for use as product labels', project for Master of Science (Natural Resources and Environment) at the University of Michigan, Ann Arbor, Michigan.

AICCM 2018, 'AICCM environmental guidelines for Australian cultural heritage collections (2018)', in Pagliarino, A 2019, Environmental guidelines, AICCM, 26 May, accessed 10 September 2019, <https://aiccm.org.au/about/who-we-are/advocacy/environmentalguidelines-australian-cultural-heritage-collections $>$.

ASTM E96 / D96M-16 2016, 'Standard Test Methods for Water Vapor Transmission of Materials', ASTM International, West Conshohoken, PA.

Balloffet, N \& Hille, J 2005, Preservation and conservation for libraries and archives, American Library Association, Chicago.

Bellotto, M, Gualtieri, A, Artioli, G \& Clark, SM 1994, 'Kinetic study of the kaolinite-mullite reaction sequence part I: kaolinite dehydoxylation', Physics and Chemistry of Minerals, vol. 22 , no. 4, pp. 207-214.

Braungart, M \& McDonough, W 2002, Cradle to cradle: remaking the way we make things, Melcher Media, New York.

Britt, KW 2012, 'Papermaking: paper properties and uses', Encyclopaedia Britannica, accessed 28 August 2019, <https://www.britannica.com/technology/papermaking/Paper-properties-anduses>

Chércoles Asensio, R, Gómez, R, San Andrés Moya, M \& de la Roja, JM 2009, ‘Analytical characterization of polymers used in conservation and restoration by ATR-FTIR spectroscopy', Analytical and Bioanalytical Chemistry, vol. 395, pp. 2081-2096. 
Chew, CH, Gan, LM \& Scott, G 1977, 'Mechanism of the photo-oxidation of polyethylene', European Polymer Journal, vol. 13, pp. 361-364.

Derrick, MR, Stulik, D \& Landry, JM 1999, Infrared spectroscopy in conservation science, The Getty Conservation Institute, Los Angeles.

Elliott, HA \& Stadmeyer, LE 2012, 'Stone paper: an overview of its characteristics and the impact they may have on forensic document examinations', Journal of the American Society of Questioned Document Examiners, June, vol. 1, no. 1, pp. 11-29.

Ezcurra, M 2012, 'Terraskin ${ }^{\circledR}$ the paper made from stone: a study of a new writing support for forensic purposes', Forensic Science International, vol. 220, pp. 164-172.

Feist, WC \& Hon, D.N-S 1984 'Chemistry of weathering and protection', in Rowel, RM (ed), The chemistry of solid wood, American Chemical Society, Washington D.C., pp. 401-454.

Gaia-kraft 2015, Stone paper brief explanation, video, YouTube, 24 August, viewed 12 July 2017, $<$ https://www.youtube.com/watch?v=ykVIkXyq9hQ $>$.

Grant, MS 2000, 'The use of ultraviolet-induced visible fluorescence in the examination of museum objects, part II', Conserve O Gram, vol. 10, no. 1, pp. 1-4.

Heart Stone Paper c. 2017, 'Products', Heart Stone Paper, accessed 16 July 2017, $<$ http://heartstonepaper.com/products $>$

Hol, D 2013, Stone paper S-Eco (RP) sustainability analysis, report for Gaia-Concept BV, The Netherlands, accessed 17 July 2017, <http://stone-paper.nl/downloads/EN\%20Taiwan\%20$\% 20$ Sustainability\%20Stone\%20Paper\%20report.pdf $>$.

Hsieh, Y-C, Lee, K-K, Cheng, S-Y \& Kao, C-C 2013, 'Offset printing quality characteristics of rich mineral paper', Applied Mechanics and Minerals, vol. 262, pp. 320-323.

Hubbe, MA \& Gill, RA 2016, 'Fillers for papermaking: a review of their properties, usage practices, and their mechanistic role', BioResources, vol. 11, no. 1, pp. 2886-2963.

Hubbe, MA 2016, 'Paper or plastic? Yes, but not as a mixture', BioResources, vol. 11, no. 3, pp. $5656-5657$.

Hunter, D 1978, Papermaking: the history and technique of an ancient craft, Dover Publications, Inc., New York.

Hustle Stone Paper 2016, 'Hustle: the world's first planner made from stone paper', Kickstarter, accessed 16 July 2017, <https://www.kickstarter.com/projects/hustle/hustle-the-worlds-firstplanner-made-from-stone-pa $>$.

Ikin, T 2013, 'Like a rock', Watercolor Artist Magazine, June, accessed 12 July 2017, $<$ http://www.taylorikin.com/articles/article_43.htm>. 
Karlovits, M \& Gregor-Svetec, D 2012, 'Durability of cellulose and synthetic papers exposed to various methods of accelerated aging', Acta Polytechnica Hungaria, vol. 9, no. 6, pp. 81100.

Karst Stone Paper 2018, 'Karst stone paper fact sheet', Karst Stone Paper, accessed 14 June 2018, $<\mathrm{https}: / /$ karststonepaper.com/ $>$.

Katz, S 2017, 'Synthetic paper', Label \& Narrow Web Magazine, January/February, pp. 66-72.

Kuypers, C 2016, 'Preliminary deterioration study of synthetic papers', minor thesis, Grimwade Centre of Cultural Materials Conservation, The University of Melbourne, Melbourne.

Larson, M 2006, 'Synthetic paper makes inroads', Converting Magazine, vol. 24, no. 10, October, pp. 32-35.

Lavédrine, B, Fournier, A \& Martin, G (eds) 2012, Preservation of plastic artefacts in museum collections, Comité Des Travaux Historiques Et Scientifiques, Paris.

Lin BT (林柏廷) 2011, Piggy Looked for the Rain (小猪找雨), Little Rainbow Educational (彩虹種 子), Taiwan.

Lu, Z, Wang, Q \& Wang X 2011, ‘A green paper tiger?', Chinadialogue, 14 March, accessed 15 July 2017, <https://www.chinadialogue.net/article/show/single/en/4233-A-green-paper-tiger$>$

Mammosa, T 2013, 'Stone paper in art!', Parax: paper made of stone, blog post, 11 September, accessed 16 July 2017, <https://papermadeofstone.com/2013/09/11/stone-paper-in-art>.

Manifesto Brands c. 2013, 'What is stone paper (Repap)?', Manifesto Brands, accessed 4 April 2017, $<$ http://www.manifesto-brands.com.au/47>.

Michalski, S 2018, 'Agents of deterioration: light, ultraviolet and infrared', Canadian Conservation Institute, 17 May, accessed 29 August 2019, <https://www.canada.ca/en/conservationinstitute/services/agents-deterioration/light.html\#det5 $>$.

Mustalish, R 2007, 'Plastics in paper: history of synthetic polymers in papermaking', in Shulla, J (ed.), Edinburgh Conference Papers 2006: Proceedings from the fifth international conference of the Institute of Paper Conservation and the first international conference of the Institute of Conservation, 26-29 July, Edinburgh, Institute of Paper Conservation, pp. 141146.

Nicomrat, D \& Tharajak, J 2017, 'A potential application of the mechanical tensile strength test for indicating paper biodegradation', Key Engineering Materials, vol. 723, pp. 183-190. 
Nuco International Ltd. 2013, Nu Stone Print information sheet, December, accessed 28 April 2017, $<$ https://www.nustonepaper.com/wp-content/uploads/2013/12/Nu-Stone-Print-InformationSheet.pdf $>$.

Parax Paper c. 2013, 'About us', Parax, accessed 15 July 2017,

$<$ http://www.paraxpaper.co.uk/Parax_Stone_Paper_About_Us_s/1670.htm>.

Pretzel, B 2008, 'Now you see it, now you don't: lighting decisions for the Ardabil carpet based on the probability of visual perception and rates of fading', ICOM Committee for Conservation, $16^{\text {th }}$ Triennial Meeting, New Delhi, vol. 2, pp. 759-765.

Reyes, JJ 2017, 'Practice playfulness: in a follow up to his May drawing board, Julio Reyes continues his exploration of the world of art materials; up this time, stone paper', Artist's Magazine, June, vol. 34, no. 5, pp. 10-13.

Slade, S 2003, 'Infosheet 3: Conservation and lighting in museums', Museums Australia Victoria, accessed 8 November 2018, $<$ https://mavic.asn.au/assets/Info_Sheet_3_Conservation_and_Lighting.pdf $>$.

The Stone Paper Co. c. 2010, 'About Rockstock', Rockstock, accessed 12 July 2017, $<\mathrm{http}: / /$ www.stonepaper.co.nz/about-rockstock>.

Stuart, BH 2007, Analytical techniques in materials conservation, John Wiley \& Sons, Chichester, England.

Taiwan Lung Meng Technology Co., Ltd. (TLM) c. 2012, ‘About us', Taiwan Lung Meng Technology Corporation Limited, accessed 24 April 2017, $<$ http://www.taiwanlm.com/page002_eng.php?ab_ID=19>.

Tang YK 2010, 'Paper from rocks: traditional papermaking techniques are being replaced by methods involving new materials', Beijing Review, 4 March, no. 9, p. 43.

Taylor, C 2018 'Géricault's “Stone Paper”, Harvard Art Museums, 25 October, accessed 23 August 2019, <https://www.harvardartmuseums.org/article/gericault-s-stone-paper>.

Zero Waste Europe 2011, 'Gaiakraft: beyond paper recycling?', Zero Waste Europe, 1 December, accessed 12 July 2011, <https:/www.zerowasteeurope.eu/2011/12/gaiakraft-beyond-paperrecycling/>. 


\section{University Library}

\section{- M M I N E R VA A gateway to Melbourne's research publications}

Minerva Access is the Institutional Repository of The University of Melbourne

Author/s:

Chu, C;Nel, P

Title:

Characterisation and deterioration of stone papers

Date:

2019-01-02

Citation:

Chu, C. \& Nel, P. (2019). Characterisation and deterioration of stone papers. AICCM Bulletin, 40 (1), pp.37-49. https://doi.org/10.1080/10344233.2019.1672951.

Persistent Link:

http://hdl.handle.net/11343/290162 